\title{
Captures
}

Figures, théories et pratiques de l'imaginaire

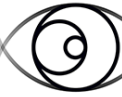

\section{Californication ou la légitimation de l'écriture télévisuelle}

C A P P Figures, théories et pratiques de limaginaire revue interdisciplinaire

\section{Sarah Sepulchre}

Volume 2, Number 1, 2017

Écrivains à l'écran

URI: https://id.erudit.org/iderudit/1059800ar

DOI: https://doi.org/10.7202/1059800ar

See table of contents

Publisher(s)

Figura, Centre de recherche sur le texte et l'imaginaire

ISSN

2371-1930 (digital)

Explore this journal

Cite this article

Sepulchre, S. (2017). Californication ou la légitimation de l'écriture télévisuelle. Captures, 2(1). https://doi.org/10.7202/1059800ar

\section{Article abstract}

La série Californication met en scène plusieurs figures d'écrivain, dont celle de son héros, Hank Moody, écrivain de génie mais manquant d'inspiration. Ces auteurs actualisent la figure du démon théorisée par Trudy Bolter en 2001. L'analyse montre que cet archétype n'est pas fondamentalement renouvelé par la série, même s'il est nuancé. Cependant, en s'alignant sur des valeurs traditionnelles liées à l'écrivain c'est à une légitimation de l'écriture télévisuelle qu'aboutit la fiction.
This document is protected by copyright law. Use of the services of Erudit (including reproduction) is subject to its terms and conditions, which can be viewed online.

https://apropos.erudit.org/en/users/policy-on-use/ 
Version enrichie de cet article : http://revuecaptures.org/node/679

\section{Californication ou la légitimation de l'écriture télévisuelle}

\section{Sarah Sepulchre}

Résumé :

La série Californication met en scène plusieurs figures d'écrivain, dont celle de son héros, Hank Moody, écrivain de génie mais manquant d'inspiration. Ces auteurs actualisent la figure du démon théorisée par Trudy Bolter en 2001. L'analyse montre que cet archétype n'est pas fondamentalement renouvelé par la série, même s'il est nuancé. Cependant, en s'alignant sur des valeurs traditionnelles liées à l'écrivain c'est à une légitimation de l'écriture télévisuelle qu'aboutit la fiction.

S'ils ne sont pas aussi récurrents que les policiers ou les médecins, les écrivains apparaissent régulièrement en tant que héros de séries télévisées. La figure archétypale est Jessica Fletcher dans Arabesque (Murder. She Wrote 1984-1996, CBS). L'auteur est d'ailleurs habituellement assimilé au détective privé, comme encore dans Castle (2009-2016, ABC) ou Bored to Death (2009-2011, HBO), qui récemment ont réactualisé ce topos. Personnages inventant des crimes parfaits dans leurs ouvrages, ils peuvent entrer dans la tête des criminels et aider à l'enquête. L'acte d'écrire en tant que tel est secondaire dans ces fictions-1.

Mais les écrivains ne sont pas absents d'autres genres télévisuels: August Booth dans Once Upon a Time (2011-, ABC), Ezra Fitz dans Pretty Little Liars (2010-, ABC Family), Lucas Scott dans Les Frères Scott (One Tree Hill 2003-2012, The WB, The CW)... Dans ce dernier cas, Lucas connaît le succès grâce à un roman qui raconte son adolescence à Tree Hill : c'est l'histoire que présente la série elle-même. Ce n'est pas le seul cas où l'auteur génère l'intrigue. La voix de Laura Ingalls encadre chaque épisode de La petite maison dans la prairie, feuilleton qui raconte l'enfance de la fillette et qui est une adaptation des ouvrages réellement créés par Laura Ingalls-Wilder. La grande œuvre criminelle de Joe Carrol, le psychopathe de The Following (2013-2015. Fox), consiste à établir un réseau de disciples " composant » chacun un chapitre de l'histoire sanglante qui constitue la diégèse de la série. Parfois, c'est le côté décalé, poétique, un peu asocial de l'auteur, qui est exploité comme terreau de croissance des personnages. C'est le cas d'Hannah Horvath dans Girls (2012-. $\underline{\mathrm{HBO}}$ ) ou de Jenny Schecter dans The L Word (2004-2009, Showtime).

Les écrivains servent donc de prétextes pour construire des personnages fantasques, pour structurer des histoires ou lancer des enquêtes criminelles. Les fictions explorent rarement la création littéraire comme telle. The Affair et Californication semblent faire exception?2. Les deux feuilletons ont pour personnage principal un écrivain; mais, si les intrigues portent sur les histoires de cœur de ces personnages, elles semblent aussi inextricablement liées à leur création. Alors on peut légitimement s'interroger à savoir si de telles séries 
télévisées, qui peuvent développer les trames narratives sur une longue durée et multiplier les personnages, permettent de renouveler la représentation de l'écrivain à la télévision?

\section{La figure de l'écrivain américain ${ }^{3}$}

Plus de 250 films américains mettent en scène des écrivains, selon le décompte de Trudy Bolter, pour qui ce foisonnement s'explique par la fascination pour les auteurs qu'entretient depuis toujours la culture américaine (Bolter, 2001: intro., §2). Les personnages représentés forment un « ensemble d'images, ouvert et fluide [...][,] un "musée" dont les murs se déplacent constamment en fonction des idées fraîches qui complètent les présupposés déjà admis » (Bolter, 2001: chap. 1, §9). Trudy Bolter distingue plusieurs archétypes. Elle entrevoit une première séparation entre les figures extrêmes (actualisées dans deux opposés : le héros et le démon) et les allégories de l'apprivoisement (incarnées par le savant simplet et l'esthétique populiste). Nous allons successivement développer ces types.

Au rayon des extrêmes, on trouve les héros et les démons. Leshéros sont les « meneurs d'hommes et de femmes, des citoyens modèles pouvant servir d'exemple aux populations " (Bolter, 2001: chap. 2, §7). C'est un " Homme Littéraire, un caractère sacré » (§8), un « animal social », " couvert de lauriers » (§10), qui considère « l'Art comme ascèse » (§22). Le Zola dépeint dans le film The Life of Émile Zola (1937) entre dans le type. Edgar Poe, Jack London, F. Scott Fitzgerald ou William Faulkner sont emblématiques du second stéréotype, le démon. II est un génie à « l'imagination débordante », " soumis à des pulsions violentes 》 (§3), déterminé à « obtenir reconnaissance, succès et fortune » (§43), évoluant « aux limites de la santé mentale, de la moralité et même de la société » (§44). II est sujet aux dépendances (alcool, drogues, pulsions sexuelles). Ce type d'auteur réactive l'idée romantique que le génie créatif est proche de la maladie mentale ou des thèses psychanalytiques considérant les «motivations artistiques comme des sublimations de pulsions de l'Inconscient » (§53).

Les deux figures suivantes fonctionnent à l'opposé, aussi proches des spectateurs que les premiers sont exceptionnels (Bolter, 2001: chap. 3, §2). Le premier, le savant simplet est un être intelligent, cultivé, mais inculte en matière d'amour (§3). Le film voit ce génie adolescent passer par des rites de passage et accéder à la maturité sentimentale. Ce héros incarne foncièrement l'optimisme dans la mesure où l'auteur est présenté comme un novateur qui parvient à se dépasser (§25). L'allégorie de l'esthétique populiste montre également un parcours d'éducation : l'écrivain — raffiné, intellectuel — doit retourner à la nature en se rapprochant d'un personnage appartenant à la culture populaire. Leur association symbolise l'articulation entre les cultures savantes et populaires qui caractérise les États-Unis (Bolter, 2001: chap. 4, §2). Ce faisant, l'artiste s'intègre à sa communauté qu'il peut mieux représenter (§14-15). 
David Martens et Myriam Watthée-Delmotte soulignent que, derrière la représentation des écrivains, l'enjeu est l'image de la littérature elle-même, l'identité de cette dernière étant « foncièrement corrélée à celle de son principal et plus visible agent de production » (7). Elle est un discours auto-constituant, mais aussi hétéroconstituée par des discours tenus sur elle. Se pose alors une question fondamentale : «Quelles peuvent être les fonctionnalités de ces figurations selon l'économie et la dynamique spécifique de ces domaines de production? » (11).

\section{Représentations, personnages et séries télévisées}

Pierre Glaudes et Yves Reuter (1996 et 1998) considèrent que le personnage est analysable selon trois niveaux : il est un marqueur typologique (qui définit le genre du texte); un organisateur textuel (son action liquide le manque initial); le lieu de l'investissement psychique et social (l'élément qui incarne la doxa 4 ). L'exploration que nous proposons dans cet article se concentre sur ce troisième niveau en tentant de déceler quel stéréotype de l'écrivain est activé par Californication.

Ce type d'étude est, selon nous, nécessairement développé en deux temps. La première phase consiste en une récolte des éléments constitutifs des personnages, la qualification et la fonctionnalité (Hamon, 1977: 154158; 1983: 185-186) ou l'« être », le « dire », le « faire » et l'« éthique » du personnage (Horvath, 1998). Dans un second temps, il s'agit d'organiser les items afin de distinguer les axes fondamentaux constitutifs des personnages 5 . «Car tous les traits sémantiques qui constituent un personnage, ou un ensemble de personnages, n'ont certainement pas le même "rendement" narratif [...] » (Hamon, 1983: 185).

Les outils inventés par Philippe Hamon semblent bien adaptés aux héros de séries car ils permettent d'analyser le système des personnages. Or, les fictions télévisuelles emploient des constellations complexes $\underline{6}$ où les héros sont multiples et sont accompagnés de figures secondaires plus ou moins récurrentes (Sepulchre, 2007). Ces configurations alambiquées ont des conséquences sur les représentations véhiculées. Il est généralement admis que le personnage principal est celui qui porte les valeurs fondamentales (Hamon, 1977, 1984). La plupart des récits proposent une thèse unificatrice qui se révèle au moment du dénouement, par la victoire du héros, grâce à ses compétences et actions. La multiplication des personnages permet de proposer des textes polysémiques où plusieurs points de vue sont présentés sans qu'un soit plus valorisé que les autres (Glaudes et Reuter, 1996: 109). Les séries télévisées ont donc la possibilité de proposer plusieurs stéréotypes d'une même figure et ainsi renouveler les représentations. D'autant que les personnages peuvent évoluer au cours d'une fiction. Parce que les protagonistes « apprennent au gré des situations auxquelles ils sont confrontés » (Perreur: 96), la durée de la narration est effectivement un élément dont il faut également tenir compte au moment de les étudier. 
Ces caractéristiques font des séries des récits particulièrement liés à la société qui les produit. Nathalie Perreur considère qu'elles ont la possibilité d'exposer la complexité du monde social en multipliant les perspectives sur un même problème : «Devant cette diversité de points de vue développés et analysés de manière critique, il revient au téléspectateur, doté d'un panorama général des possibles, de se faire le sien [...]. » (Perreur: 95.) Les séries contemporaines jouent ainsi un rôle dans la construction d'une critique sociale $\underline{7}$. Sabine ChalvonDemersay (2015) avance même que l'on peut tenir le héros responsable des positionnements politiques pris par les fictions, étant donné qu'il est l'élément où les valeurs s'activent. Que nous disent, dès lors, les protagonistes de Californication? La série renouvelle-t-elle la figure de l'écrivain?

\section{Hank Moody, écrivain non pratiquant}

Selon les dires de plusieurs personnages durant l'épisode pilote, Hank Moody est un écrivain à succès. Karen, son ex-femme, voit en lui un talent incroyable. Mia le taxe d' " auteur célèbre ». Ses amis, Charlie et Marcy, le présentent comme un " grand écrivain ». Meredith, rencontrée lors d'un souper avec des amis, considère qu'il doit être fier. Cette caractérisation sera rappelée régulièrement durant les sept saisons de Californication. Un élève souligne qu'il est un « super écrivain » (s3e2). Plusieurs se déclarent fans. Atticus Fetch le décrit comme suit : « Baudelaire, Bukowski et Oscar Wilde condensés en un putain de talentueux écrivain. » (s6e1.) Richard Bates, un autre écrivain, lui fait remarquer qu'il est particulièrement bon dans son domaine (s6e4).

Pourtant, il s'agit d'un auteur en crise. Dans la première scène, onirique, Hank avoue qu'il ne sait plus écrire. Une confession répétée plusieurs fois durant le pilote. « J'ai eu de la veine pour un seul livre », remarque-t-il (s1e1). Or, Hank considère que l'écriture est un élément primordial. «La seule chose qui fait de toi un écrivain, c'est t'asseoir sur une chaise et transcrire ce qu'il y a dans ta tête sur le papier ", déclare-t-il à Becca (s6e10). Si on le suit dans cette définition, il n'est effectivement plus un auteur au moment où commence la série. L'épisode se termine d'ailleurs sur le héros qui s'installe devant son ordinateur ne parvenant à écrire que : « Fuck ». Cela l'amène à se présenter plus tard comme un écrivain non pratiquant (s1e2). Le manque d'inspiration dure depuis plusieurs années (selon Charlie, s1e2; et Mia, s1e3).

Le génie est la dernière facette de la figure d'écrivain qu'incarne Hank Moody. II s'agit d'un don inné. Karen a la conviction qu'il est destiné à l'écriture (s1e3). Faith considère qu'il possède « cette chose difficile à décrire, cet éclair, cette étincelle » qu'elle refuse d'appeler du génie, le mot étant trop galvaudé. Elle poursuit en affirmant qu'il fait exactement ce qu'il était destiné à faire (s6e5). Rick Rath le surnomme le « talent » la première fois qu'il le rencontre (s7e1).

\section{Une série, des écrivains}

Cet auteur de génie, célèbre, mais en manque d'inspiration constitue l'épicentre de Californication. II n'en est 
cependant pas la seule figure d'écrivain. Quinze personnages sont des « writers ». Le mot américain signifiant autant « écrivain », « journaliste », « essayiste », « scénariste », " parolier », il n'est pas étonnant de retrouver dans cette liste des auteurs de livres de développement personnel comme Julian (saison 2) et Ophélia Robins (saison 6), ainsi que Sue Collini (saison 3) ou Krull (saison 6), qui écrivent leurs mémoires. Hank rencontre David Wilder, romancier et professeur (saison 3). Tyler est un scénariste de cinéma (saison 5), Atticus Fetch une star du rock qui compose une comédie musicale (saison 6). Plusieurs personnages signalent qu'ils ont écrit un roman (Nikki, s1e4; le professeur de Mia, s1e5; l'avocate de Hank, s4e5). Néanmoins, la fiction ne s'attarde généralement pas sur ces personnages. Leur fonction consiste avant tout à marquer le fossé les séparant du vrai romancier, Hank Moody.

Quatre d'entre eux offrent des figures plus intéressantes. Tout d'abord, Mia Lewis (dont le nom de plume est Mia Cross) n'est pas véritablement une romancière dans la mesure où elle vole le roman de Hank. Mia fonctionne en creux : tous les compliments qu'elle reçoit sont imputables à Hank. Karen la déclare un prodige (s2e4). Son livre décroche la meilleure critique que Hank ait jamais eue selon Charlie (s2e11). Souvent ces éloges vont de pair avec des critiques à l'égard de Hank. Dani, l'agent de Mia, se demande ce qui lui est arrivé, car il avait du talent (s1e10) — il faut évidemment noter l'utilisation du passé. Une journaliste du Rolling Stone qui trouve les livres de Hank « puérils, grossiers, adolescents comme lui » (s2e6) apprécie au contraire l'écriture de Mia (s2e11) et le déclare « has been » (s2e11). Mia finira toutefois par être démasquée. Quand elle compose quelques pages pour la version de poche, son écriture est décousue et prétentieuse, selon son gérant (s3e12).

Les trois personnages suivants servent de miroir au héros. Becca, la fille de Hank, annonce qu'elle quitte l'école pour entamer une carrière d'écrivain (s6e1). Elle s'estime, elle aussi, destinée à exercer ce métier : toute sa vie l'y a menée, déclare-t-elle, ajoutant qu'elle est la fille de son père, qu'elle le veuille ou non (s6e1). Quand elle doute, Hank lui rétorque qu'elle est née pour faire ce qu'elle fait (s6e8). Mais le talent les distingue. Hank critique sa première ébauche tout en avouant à Karen qu'elle est meilleure que lui à son âge (s6e8). Becca doit donc s'améliorer, elle n'est qu'une écrivaine en devenir. Les excès sont un autre trait qu'ils partagent. Sa relation à l'alcool, aux drogues et au sexe n'est pas aussi maladive que celle de son père, mais elle se teste malgré tout.

Quand il rencontre Richard Bates, Hank lui annonce qu'il est un fan (s3e1) et Richard en retour propose un toast à la page blanche. Le manque de créativité, mais aussi l'excentricité, l'abus d'alcool et l'amour de Karen les rassemblent. Ils partagent également une même vision de l'écriture défendant, à force de métaphores sexuelles, l'idée qu'on ne peut écrire que sur ce qu'on connaît. Hank déclare à ses élèves : « Je ne peux pas vous apprendre à écrire [...], je ne peux qu'écrire que sur ce qui m'excite, ce qui me fait bander » (s3e2). 
Richard proclame : «C'est pourquoi il est si important de vivre d'abord et d'écrire ensuite. Stylo, papier, portable... Ces merdes n'ont aucune importance. Le seul outil dont tu as besoin est juste là entre tes jambes. [...] Suis tous tes instincts. » (s6e11.) Cependant, Richard est plus avancé dans sa carrière. Karen, provocante, déclare à Hank que Richard lui ressemble, mais en plus génial (s5e1). Ceci permet à Richard de lui donner des conseils.

Rick Rath, dernier des doubles du héros, occupe la fonction deshowrunner $\underline{8}$ de la série télévisée pour laquelle Hank travaille dans la dernière saison. À l'occasion de leur première rencontre, l'écart entre les deux hommes semble total. Rick ne cherche pas nécessairement le talent à l'état pur, mais quelqu'un capable d'être à l'heure, gentil avec les autres, d'accepter les remarques, de travailler avec autrui (s7e1). Rick se comporte en meneur d'équipe et on sent que l'écriture télévisuelle est un art collectif. Pourtant, les deux hommes présentent certaines similitudes. Rick a habité New York et il était alors écrivain. II déclare à Hank que ses ouvrages lui rappellent ce qu'il écrivait alors (s7e1). Au fil de la saison, Rick se révèle à la fois lucide vis-à-vis de Hank (il comprend qu'il pensait abandonner, s7e2), capable de lui faire accepter les règles de travail de la télévision (s7e2, s7e3), de le conseiller subtilement (s7e8) et de reconnaître son talent (s7e7). Quand la série est annulée, il l'enjoint de créer son propre feuilleton à partir de ce qu'il connaît (s7e11).

Si Hank donne le ton, on voit la cohérence de ces portraits croisés d'auteurs secondaires. Ceux qui se distinguent sont des prodiges, dont le talent est inné et reconnu, qui souffrent régulièrement de crise de la page blanche et dont l'art possède un aspect sexuel. Plusieurs personnages semblent reconstruire la vie d'un écrivain depuis les premiers brouillons (Becca), les premiers succès (Hank), la consécration (Richard) ou la diversification (ici vers l'industrie télévisuelle, Rick). Si Californication met en scène plusieurs écrivains, ce n'est pas pour souligner la diversité, mais pour sublimer le mythe du génie à l'état pur. Les personnages de Californication relèvent donc de l'archétype du démon répertorié par Trudy Bolter. Hank fait irrémédiablement penser au Charles Bukowski décrit par François Bovier (2010). C'est un « dur » qui « multiplie les provocations » (47), adopte une « position hautaine » (53), « affirme son égocentrisme et son incivilité » (53) : il boit, multiplie les rencontres et s'autodétruit (54), « un écrivain à scandale dont la reconnaissance est d'emblée internationale » (54). La série nuance un peu cette caractérisation primordiale, nous allons le voir; mais sans jamais véritablement la remettre en question. La fiction n'emploie pas le potentiel du système élargi de personnages afin de proposer des représentations polysémiques.

\section{Écriture}

Face à cette omniprésence des personnages d'écrivain, l'absence presque totale de l'écriture à l'image étonne. Les seules fois où Hank Moody s'installe devant son ordinateur (s1e1, s1e6, s1e7, s1e8) ou sa machine à écrire (à partir de la saison 3), il s'agit d'une courte séquence terminant un épisode et, généralement, il ne crée 
pas vraiment (s1e1, s1e6, s6e5). À la fin du pilote, il ne dactylographie que « Fuck ». Parfois, il boit (s1e8) ou il fume (s5e9). Cela ne signifie pas qu'il ne rédige rien; au contraire, au fil des saisons, il termine le roman que Mia lui vole (s1e9), une biographie (saison 2), une autre fiction refusée par tout le monde (s3e1), un scénario (s4e3), une chanson (s5e6), le script d'une comédie musicale, un épisode de série télévisée (s7e4).

Seulement, on le voit soit commencer à écrire (s4e3), soit retirer la dernière page de la machine et s'adonner à son rituel : siroter un verre de whisky (s2e12, s5e3, s6e8). L'écriture comme telle reste hors champ. C'est d'autant plus singulier qu'elle est lourdement présente dans la caractérisation de Hank, nous venons de le voir, et qu'il est régulièrement rappelé qu'un écrivain... écrit.

Un homme martelant un clavier n'est probablement pas l'action la plus haletante à filmer. La télévision ne diffère pas en cela du cinéma, dont plusieurs auteurs ont souligné la difficulté de représenter cette activité (Boillat: 10; Bolter, 2001: chap. 2, §5). Ce n'est pourtant pas non plus une séquence impossible à montrer $\underline{9}$. Par exemple, dans Sex and the City, la journaliste Carrie Bradshaw est souvent vue en train de travailler. Ces scènes ne durent pas longtemps et les plans sont appuyés par la lecture de l'article en composition en voix off. L'image en tant que telle ne semble donc pas se suffire à elle-même. Cela dit, la faible télégénie de l'acte d'écriture n'explique pas tout. En effet, des épisodes entiers de sitcom ou de soap opera se bornent à l'exposition de dialogues (par exemple Friends, Big Bang Theory ou Les feux de l'amour). Les "séries collèges $\underline{10}$ », les sagas familiales ne brillent pas non plus par la flamboyance de leurs actions. En tout état de cause, la question télégénique ne justifie pas une telle absence au sein d'une fiction à ce point centrée sur des personnages d'auteur.

Car, si on peut comprendre que la phase d'écriture ne soit pas représentée, il est étonnant qu'elle ne soit pas non plus intégrée dans les trames narratives. Hank ne fait jamais allusion à sa création, il n'explique pas les difficultés rencontrées, il ne décrit pas les idées qui émergent. Alors qu'une série déploie ses trames sur la durée, Californication n'en profite pas pour montrer le travail en cours. Quand Hank écrit, c'est de manière ponctuelle, entre deux épisodes 11 .

Quelques rares contre-exemples démontrent que c'est cependant possible. Hank doit composer les paroles d'une chanson pour Kali (s5e6). La chanteuse alors évoque ses souvenirs, un amour perdu parce qu'elle a souhaité tenter sa chance à Los Angeles. Plusieurs flash-backs montrent son histoire mais aussi les échos qu'elle éveille chez Hank qui a lui aussi quitté New York, où il vivait un amour idéal avec Karen. Cet épisode illustre la manière dont une inspiration naît, comment Hank se nourrit de l'expérience de Kali, même si l'écriture reste hors champ.

Une fois le scénario de Santa Monica Cops finalisé, le tournage commence et Hank (s5e9) doit rester disponible pour les réécritures de dernière minute. Si on ne le voit pas plus écrire que d'habitude, la durée de 
l'épisode correspond à celle du travail. Hank est assis dans sa caravane, devant son ordinateur et reçoit plusieurs visites (celle de l'acteur principal, celle de l'actrice, celle du producteur, etc.). L'écriture se déroule sur la longueur et non dans l'ellipse entre deux séquences.

La dernière saison présente un exemple beaucoup plus étendu. Hank intègre l'équipe de scénaristes de la série télévisée adaptée de Santa Monica Cops (s7e1). On assiste aux réunions des auteurs (s7e2), à l'échange de perspectives sur les scénarios (s7e2). Rick lui attribue l'écriture de l'épisode 3 et lui demande de présenter ses idées sur les courbes narratives (s7e3). Hank reçoit des remarques et la réécriture collective commence (s7e7). Il doit ensuite gérer le tournage de l'épisode qu'il a composé (s7e8). De nouveau, la phase d'écriture reste hors champ (s7e4) et les moments de réunion avec l'équipe de scénaristes restent euphémisés. Cependant, le processus de création est davantage mis de l'avant et les possibilités offertes par la sérialité se trouvent exploitées, puisque le téléspectateur voit le travail avancer.

II revient à Becca de faire l'objet d'un récit où la progression de l'écriture est intégrée dans la longueur. La sixième saison est consacrée à ses débuts littéraires. La jeune femme annonce qu'elle abandonne l'école (S6e1) et qu'elle travaille à son premier roman (s6e2). Elle pose des questions à Richard Bates (s6e4). Son manuscrit approche des 300 pages dans l'épisode 6 et elle déclare écrire tous les jours. Il est terminé l'épisode suivant et elle le donne à lire à Hank (s6e7), qui lui présente ses critiques dans l'épisode 8. De nouveau, l'écriture comme telle n'est pas filmée, mais le processus de création est étendu, discuté, mis en scène.

Cette ambivalence de la série télévisée — donnant à la fois de l'importance à l'écriture dans la caractérisation des personnages et rendant invisible l'acte de création - entoure l'écriture d'un voile de mystère. L'acte d'écrire qui définit le génie des écrivains est tellement sacré qu'il demeure irreprésentable. La télévision rejoint ici le cinéma, où « la genèse de l'œuvre littéraire constitue une sorte de point aveugle de la représentation » (Boillat: 13). C'est d'autant plus surprenant que, à certains égards, Californication se démarque. En effet, Rachel Noël, dans un article sur la figure de Virginia Woolf, remarque que, dans la plupart des biopics, les artistes sont des « génies individuels, travaillant de manière passionnée, instinctive, quelquefois même sans effort [...] » (Noël: 78). Or, dans la série, l'acte de création est souvent collectif : c'est la confrontation des passés de Hank et Kali qui donne naissance à la chanson, c'est la transmission de l'expérience du père qui permet à la fille auteure de progresser, c'est la discussion entre les scénaristes qui fait émerger les idées de scénarios. Par ailleurs, les parcours de Becca (qui débute) et de Hank (qui s'initie à l'écriture télévisuelle) suggèrent qu'on peut apprendre à écrire. II s'agit là d'un paradigme très américain, estime Trudy Bolter 12 , néanmoins beaucoup moins fréquent que celui, très romantique, du génie inné (massivement investi en Hank). La série apporte donc quelques nuances aux stéréotypes mais pas autant qu'on pouvait l'attendre.

\section{Énergie sexuelle, énergie créatrice}


Si l'écriture demeure hors champ, que montre alors Californication? La vie de Hank Moody. Ce n'est pas anodin pour une série où le conseil récurrent est d'écrire sur ce qu'on connaît. Or, le quotidien de Hank, ce sont ses frasques sexuelles. Sur le site officiel de la chaîne, Hank est présenté comme ayant tous les vices : alcool, drogues et frénésie sexuelle. Énergie sexuelle et créatrice se confondent en lui. C’est parce que Karen couche avec lui durant la première saison qu'il parvient à écrire un roman (s1e9). Faith, dans une moindre mesure, joue ce rôle dans la saison 6 . Hank la définit comme une groupie, elle le reprend : « Je préfère muse. [...] Muse est plus approprié car on a écrit des chansons sur moi » (s6e2).

Le sexe constitue par ailleurs une caractéristique de son style, aux yeux de plusieurs personnages. Karen lui fait remarquer que toute l'esthétique de son écriture tourne autour de ce thème (s5e8). Charlie déclare que ses mots « font bander » (s4e4). Lui-même utilise cette métaphore pour qualifier son travail (voir l'extrait cité plus haut, s3e2). Hank établit un clair parallèle entre sexe et fiction, qui possèdent une structure en trois actes : un début, un milieu et une fin, avec une surprise au troisième acte ( $55 \mathrm{e} 4$ ). Becca, en bonne apprentie, annonce qu'elle écrit un roman sur la vie, l'amour, le sexe et la mort (s6e2).

Californication rapproche le sexe de l'écriture, l'énergie sexuelle de l'énergie créatrice, rejoignant par là les représentations traditionnelles au cinéma. Alain Boillat souligne que les réalisateurs, obnubilés par l'angoisse de la page blanche, restaurent « symboliquement la "fertilité" de l'auteur » par le sexe (Boillat: 22). Et cet héritage est, de nouveau, celui de l'archétype du démon, sauf à considérer qu'au fil des saisons, Hank doit apprendre à aimer. En effet, ses activités sexuelles débridées sont en contradiction avec sa vie sentimentale tout entière consacrée à Karen, dont il est éperdument amoureux. On pourrait y déceler une hybridation entre les figures du démon et du savant simplet; cependant cela reste une nuance relativement ténue apportée par les intrigues.

Dans Californication, le sexe constitue à la fois la caractéristique de l'écrivain, ce qui le nourrit, son sujet favori, mais aussi ce qui alimente l'image. On reconnaît ici le réflexe de certains films assimilant le texte au protagoniste (par exemple, Kafka est représenté dans une intrigue kafkaïenne). Les scènes érotiques conviennent particulièrement bien à l'esthétique « moodienne ». Les écrivains de la série réactivent l'idée qu'auteur et œuvre se confondent, un phénomène connu depuis le romantisme (Bolter, 2001: chap. 1, §95) et largement réactivé au $X X^{e}$ siècle avec des auteurs ultra-médiatisés comme Hemingway ou J. D. Salinger (Bolter, 2001: chap. 1, §56, §82).

\section{Le monde de l'art}

Au-delà de l'entourage immédiat de Hank, Californication dévoile aussi le microcosme dans lequel baigne le héros. Comme l'écriture reste hors champ, le monde de l'édition littéraire est absent ou présent sous forme 
uniquement anecdotique. On voit Hank donner une conférence à une seule reprise (s1e2). C'est grâce au personnage de Mia que ce milieu est introduit : on la voit négocier avec son agent (s1e10), puis avec une éditrice (s1e11). Elle promeut son livre (s2e9, s2e11) et offre une lecture publique dans une librairie (s3e12). Toutefois, globalement, ce champ demeure aussi délaissé que l'acte de création lui-même.

En revanche, Hollywood apparaît dans chaque épisode. Hank rencontre des rédacteurs en chef (s1e2), des agents (s5e10), participe à des mondanités (s1e4), répond à la presse (s1e5), négocie avec le milieu cinématographique (s1e7, s4e1, s4e2, s4e4, s5e1, s5e9, s6e4), traite avec l'auteur d'une comédie musicale (s6e1) et s'intègre à l'équipe d'une série télévisée (saison 7). On traite d'ailleurs Hank souvent comme une star du rock. Un journaliste compare l'attente de son prochain livre à celle de l'hypothétique retour des Guns ' $n$ ' Roses (s1e5). Lew Ashby souligne que son écriture a " une énergie très rock 'n' roll » (s2e2). Dans la saison 6 , Faith, pourtant muse de musicien, accepte de s'occuper de son cas. Or, Hank Moody exècre le star system.

Tout le propos de Californication consiste à établir une distinction nette entre les différents arts. Selon Karen, seule la littérature est un art pour Hank (s1e7). Celui-ci considère qu'il est entouré de scribouillards : « Je galvaude pas ce mot, moi. Tu les écoutes, ils sont tous "écrivains", ici. » (s1e11.) Cette opposition est symbolisée par deux lieux : New York et Los Angeles. New York représente un paradis perdu, le lieu où Hank écrit des romans. Pour Hank, il était écrivain quand il habitait « un petit bled appelé New York » (s1e1). II ne parvient pas à écrire à Los Angeles, lieu de déperdition symbolisant l'argent sale. "À la minute où j'ai pris l'argent, mes lèvres se sont emparées de l'érection géante qu'est l'industrie du cinéma et l'ont sucée, comme une pro. »(s1e2.)

En conséquence, la trame narrative de Californication amène, par différents procédés, cette idéalisation de la littérature. La manière dont le héros est caractérisé, le positionnement respectif des personnages et la distinction de ceux bénéficiant d'un talent inné; la création d'un voile de mystère sacré autour de l'écriture et du monde de l'édition; l'attention portée à l'énergie sexuelle, tout concourt à dresser le portrait d'un génie démoniaque, d'un art sublime qu'on ne peut qu'opposer à Hollywood et à sa clique de fausses vedettes. Les nuances apportées - la recherche sentimentale qui pourrait établir un lien avec le savant simplet ou les quelques endroits où le travail de création intègre sa dimension collective ou sa durée (qui ouvre la possibilité à l'apprentissage) - ne sont pas assez systématiques pour véritablement remettre en question ce constat.

La septième saison, autant dire la conclusion, de Californication dès lors surprend. Elle se déroule dans le monde de la télévision qui, par certains aspects, semble aussi détestable que celui du cinéma. Hank ne la regarde d'ailleurs pas (s7e1). Les scénaristes sont incompétents, Rick Rath espionne ses auteurs (s7e1), l'inactivité semble régner (s7e3), l'organisation (horaire, hiérarchie, relations entre personnes) l'assimile à un « job de salarié » (s7e2). Cependant, d'autres éléments distinguent ce média. Rick Rath est un personnage 
proche de Hank. Le travail d'écriture de la série est très différent de celui du cinéma, où un script stéréotypé suffit. Les scénaristes partent de leurs expériences. Rick révèle que c'est ainsi que la télévision fonctionne : «Les gens confessent leurs plus profonds et noirs secrets, on les passe au travers d'un prisme et on les met sur écran » (s7e2). Une série s'écrit donc comme un roman, en partant de ce qu'on connaît. Et c'est exactement le principe que rappelle Rick lorsqu'il conseille à Hank de créer sa propre série (s7e11).

Après avoir construit l'image d'un Auteur (avec la majuscule du génie) pendant six saisons, la fiction offre donc un rebondissement spectaculaire dans la septième. C'est à cette occasion que Californication apporte sa nuance la plus importante à l'archétype du démon. Rick Rath ressemble à cet acolyte dépeint par Trudy Bolter qui amène l'écrivain à considérer l'art populaire et à se l'approprier (Bolter, 2001: chap. 4, §2). En dernier recours donc, Californication développe l'allégorie de l'esthétique populiste et renoue avec cette dualité qui, selon Trudy Bolter, a toujours traversé l'écriture aux États-Unis, où « les différents niveaux de culture ("high" la culture savante - et “low") n'ont jamais cessé de s'interpénétrer » (Bolter, 2001: chap. 1, §100). Cette ultime saison nous permet également d'apporter une réponse à la question posée par David Martens et Myriam Watthée-Delmotte. En plaçant sur un même pied création littéraire - sacrée - et écriture télévisuelle, écrivain et scénariste, c'est bien à une légitimation des séries qu'aboutit Californication. Comme la littérature, la télévision est un discours auto-constituant, et son argumentaire est ici plutôt évident.

1. Même si, par exemple, Richard Castle écrit et publie des ouvrages au fil des saisons.

2. Cet article sera centré sur Californication. Pour une analyse de The Affair, voir l'article de Nadja Cohen dans ce même numéro.

3. Pour une version illustrée et grand public de ce paragraphe, voir l'émission Blow Up d'Arte.

4. Autrement dit «le back-ground idéologique d'une société à partir duquel s'organise [...] l'ensemble "mou" des évidences partagées, [...] des stéréotypes » (Glaudes, 1996: 109).

$\underline{5}$. $\quad$ Analyser le système des personnages d'un texte, c'est donc opérer une série de réductions et de tris : d'une part passer de la multitude des actions et des modalités lexicalisées [...] au plus petit nombre des déterminations cardinales [...][,] d'autre part à un niveau plus abstrait, de rôles actantiels constitués à partir des trois modalités du vouloir, savoir, et du pouvoir. " (Hamon, 1983: 185-186.)

6. La multiplication du nombre des personnages n'est qu'un aspect de la complexification des récits. On constate aussi l'augmentation du nombre des trames narratives, l'hybridation des formes, l'apparition de la réflexivité (Lotz; Mittel; Sohet; Newcomb).

7. Eric Macé a régulièrement défendu ce point de vue (2005). Il considère que les médias forment un champ où l'on peut saisir les conflits qui animent la sphère publique; les imaginaires collectifs possèdent donc une dimension politique (2005).

8. Le showrunner est celui qui a eu l'idée de l'histoire et qui écrit le pilote. II a la charge de la direction artistique et de la production de la fiction (choix des acteurs, supervision de l'écriture, du tournage et du montage des épisodes).

9. Pour des contre-exemples cinématographiques plus riches, voir les contributions de Baptiste Villenave et de Mathilde Labbé au présent dossier.

10. Les séries collèges sont, selon Dominique Pasquier, « des séries de fiction télévisuelle qui mettent en scène des adolescents dans le cadre scolaire ou universitaire » (Pasquier, 1997: 815). Elle cite en exemples Hélène et les garçons, Premiers Baisers, Sauvés par le gong ou Beverly Hills.

11. II écrit Fucking \& Punching entre les épisodes 8 et 9 de la saison 1. II rédige la biographie de Lew Ashby entre les épisodes 11 et 12 de la saison 2. II a terminé un autre roman avant que la saison 3 ne débute. II adapte le scénario de Fucking \& Punching entre les épisodes 3 et 4 de la saison 4. II publie un nouveau livre, Californication, entre les saisons 4 et 5 . II crée le scénario de Santa Monica Cops (le film) entre les épisodes 2 et 3 de la saison 5.

12. "L'art littéraire n'y est pas considéré comme une histoire d'inspiration divine et d'élection aléatoire par le Destin, mais en termes 
d'apprentissage, de travail et de perfectionnement, comme un artisanat. » (Bolter, 2001: chap. 1, §36.)

\section{Bibliographie}

BoILlat, Alain. 2010. «Le déni de l'écrit à l'écran. L'écrivain, son œuvre et l'univers filmique».

Décadrages, no 16-17, p. 9-46. <http://decadrages.revues.org/236>.

Bolter, Trudy. 2001. Figures de l'écrivain dans le cinéma américain. Itinéraires de la " voix baladeuse"

Rennes : Presses universitaires de Rennes, 298 p. <http://books.openedition.org/pur/1802?lang=fr>.

Bovier, François. 2010. «Bukowski en abîme : de l'écrit à l'écran. À propos de "Contes de la folie

ordinaire", Marco Ferrei, et de "Barfly", Barbet Schroeder ». Décadrages, no 16-17, p. 47-70.

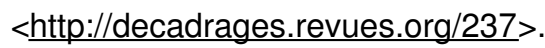

Chalvon-Demersay, Sabine. 2015. «Pour une responsabilité politique des héros de séries télévisées». Quaderni, no 88, p. 35-51.

Glaudes, Pierre et Yves Reuter. 1996. Personnage et didactique du récit. Metz : Centre d'analyse de l'Université de Metz, $221 \mathrm{p}$.

Glaudes, Pierre et Yves Reuter. 1998. Le Personnage. Paris : Presses universitaires de France, " Que saisje? », $127 \mathrm{p}$.

Hamon, Philippe. 1977. «Pour un statut sémiologique du personnage», dans Roland BARTHES, Wolfgang J

Kayser, Wayne C Booth et Philippe Hamon (dir.), Poétique du récit. Paris : Seuil, p. 115-180.

Hamon, Philippe. 1983. Le Personnel du roman. Le système des personnages dans les Rougon-Macquart

d’Émile Zola. Genève : Droz, « Histoire des idées et critique littéraire », 329 p.

Hamon, Philippe. 1984. Texte et idéologie. Valeurs, hiérarchies et évaluations dans l'œuvre littéraire

Paris : Presses Universitaires de France, $227 \mathrm{p}$.

HoRvATH, Krisztina. 1998. «Le personnage comme acteur social (les diverses formes de l'évaluation dans

"La Peste" d'Albert Camus) 》. Palimpszeszt, no 11. <http://magyar-

irodalom.elte.hu/palimpszeszt/11 szam/09.htm>.

KaPINOS, Tom. 2007 [-2014]. Californication. États-Unis : Paramount, saison 1-7, DVD, 84 ép.

Lotz, Amanda. 2007. The Television Will Be Revolutionized New York : New York University Press, 321 p.

MACÉ, Éric. 2005. «Mouvements et contre-mouvements culturels dans la sphère publique et les médiacultures », dans Éric MaIGret et Éric Macé (dir.), Penser les médiacultures. Nouvelles pratiques et nouvelles approches de la représentation du monde. Paris : Armand Colin, p. 41-66.

MARTEns, David et Myriam WatThee-Delmotte (dir.). 2012. L'Écrivain, un objet culturel. Dijon : Éditions universitaires de Dijon, $327 \mathrm{p}$. 
MITTEL, Jason. 2006. « Narrative Complexity in Contemporary American Television». The Velvet Light

Trap, no 58, automne. <http://juliaeckel.de/seminare/docs/mittell narrative complexity.pdts.

Newcomb, Horace. 1985. «"Magnum", The Champagne of TV?». Channels of Communication, vol. 5, no 1, p. 23-26.

NoËL, Rachel. 2010. «Entre génie et Ophélie. Images de Virginia Woolf dans "The Hours"». Décadrages, no 16-17, p. 71-94. <http://decadrages.revues.org/238>.

PASQUIER, Dominique. 1997. «Télévision et apprentissages sociaux. Les séries pour adolescents».

Sociologie de la communication, vol. 1, no 1, p. 810-830.

Perreur, Nathalie. 2011. «La néo-série, arène d'évaluation culturelle d'une société américaine en crise».

Réseaux, no 165, p. 83-108. <http://www.cairn.info/revue-reseaux-2011-1-page-83.htm>.

SePULCHRE, Sarah. 2007. «Le Héros multiple dans les fictions télévisuelles à épisodes. Complexification

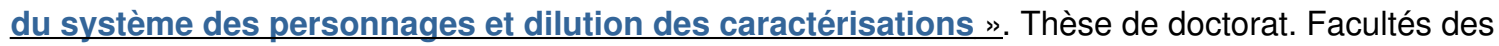

Sciences économiques, sociales, politiques et de la communication, Université catholique de Louvain,

$364 \mathrm{f}$.

SOHET, Philippe. 2007. Images du récit. Québec : Presses universitaires du Québec, 358 p. 